\title{
Machine learning in medicinal plants recognition: a review
}

\begin{abstract}
Medicinal plants are gaining attention in the pharmaceutical industry due to having less harmful effects reactions and cheaper than modern medicine. Based on these facts, many researchers have shown considerable interest in the research of automatic medicinal plants recognition. There are various opportunities for advancement in producing a robust classifier that has the ability to classify medicinal plants accurately in real-time. In this paper, various effective and reliable machine learning algorithms for plant classifications using leaf images that have been used in recent years are reviewed. The review includes the image processing methods used to detect leaf and extract important leaf features for some machine learning classifiers. These machine learning classifiers are categorised according to their performance when classifying leaf images based on typical plant features, namely shape, vein, texture and a combination of multiple features. The leaf databases that are publicly available for automatic plants recognition are reviewed as well and we conclude with a discussion of prominent ongoing research and opportunities for enhancement in this area.
\end{abstract}

Keyword: Medicinal plants; Machine learning; Leaf identification; Classification 